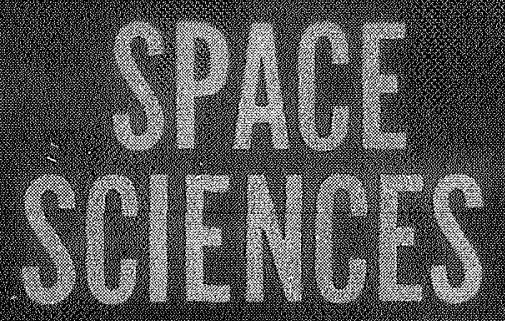

MABOMATORY

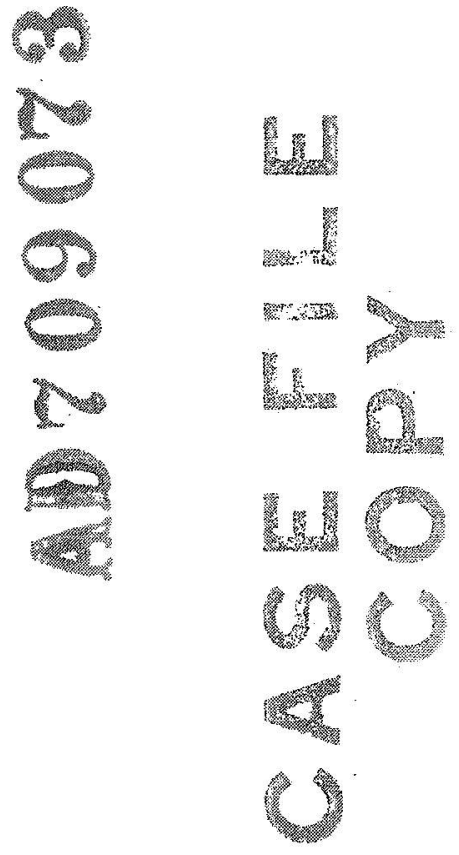

COMPARISON OF THE MEAN PHOTOSPHERIC MAGNETIC FIELD AND THE INTERPLANETARY MAGNETIC FIELD

A. Severny, J.M. Wilcox,

P. H. Scherrer and D. S. Colburn

$$
\text { M) }-1 / 6 / 9
$$

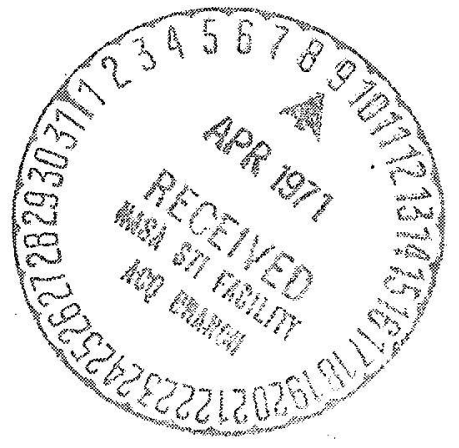

Series 11 , Issue 36

May 5, 1970

UNIVERSITY OF CALIFORNIA, BERKELEY

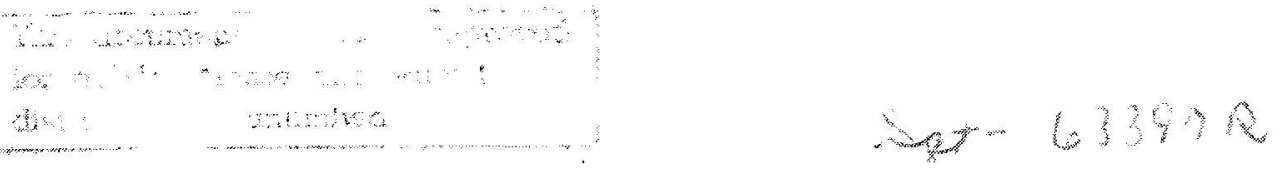


COMPARISON OF THE MEAN PHOTOSPHERIC MAGNETIC FIELD
AND THE INTERPLANETARY MAGNETIC FIELD*

A. Severny, J. M. Wilcox,

P. H. Scherrer and D. S. Colburn

\begin{abstract}
Technical Report
ONR Contract N00014-69-A-0200-1016, Project NR 021 101 NASA Grant NGL 05-003-230 and NSF Grant GA-1319
\end{abstract}

Distribution of this document is unlimited.

Reproduction in whole or in part is permitted for any purpose of the United States Government.

* Submitted to Solar Physics. 


\title{
COMPARISON OF THE MEAN PHOTOSPHERIC MAGNETIC FIELD \\ AND THE INTERPLANETARY MAGNETIC FIELD
}

\author{
A. Severny \\ Crimean Astrophysical Observatory \\ Nauchny, Crimea, USSR \\ J. M. Wilcox, P. H. Scherrer \\ Space Sciences Laboratory \\ University of California \\ Berkeley, California 94720, USA \\ and \\ D. S. Colburn \\ NASA Ames Research Center \\ Moffett Field, California 94035, USA
}

Abstract. The mean photospheric magnetic field of the sun seen as a star has been compared with the interplanetary magnetic field observed with spacecraft near the earth. Each change in polarity of the mean solar field is followed about $4 \frac{1}{2}$ days later by a change in polarity of the interplanetary field (sector boundary). The scaling of the field magnitude from sun to near earth is within a factor of two of the theoretical value, indicating that large areas on the sun have the same predominant polarity as that of the interplanetary sector pattern. An independent determination of the zero level of the solar magnetograph has yielded a value of $0.1 \pm$ 0.05 gauss. An effect attributed to a delay of approximately one solar rotation between the appearance of a new photospheric magnetic feature and the resulting change in the interplanetary field is observed. 
The magnetic field of the Sun seen as a star has been measured with the Crimean tower telescope (Severny, 1955) using the parallel beam from the coelostat mounting falling on the magnetograph slit. This method gives the mean (averaged over all the elements of the image with the distribution of the brightness as a weighting function) longitudinal field strength (for the description of the mean solar field observations see Severny, 1969). This mean solar field observed in March through June 1968 has been compared with observations of the interplanetary magnetic field obtained with the Ames Research Center magnetometers on the spacecraft Explorers 33 and 35, for which C. P. Sonett is the Principal Investigator. The spacecraft magnetometer experiments have been described by Mihalov et al. (1968). A brief report on this comparison has been given by Wilcox et al. (1969). All these papers should be consulted for further details.

The top graph in Figure 1 shows a comparison of the observed mean solar field (dots) with the polarity of the interplanetary magnetic field. The polarity of the interplanetary field was determined using the method described by Wilcox and Colburn (1969). The interplanetary data was plotted on the graph of solar observations taking account of the transit time of solar wind plasma from sun to earth, as described later in this paper. It can be seen in Figure 1 that every time (with one small exception) the mean solar field changes polarity a similar change of polarity (interplanetary sector boundary) occurs in the interplanetary field. The polarity changes in the solar and in the interplanetary observations are usually as close together in time as could be expected, since the solar observations are obtained only once per day, and since changes in the 
solar wind velocity from one sector boundary to the next could change the transit time of the boundaries by a day or more.

The bottom graph in Figure 1 shows the total flux $\mathrm{H}_{\mathrm{S}}$ (divided by the visible area of the solar disk $\pi R_{\odot}^{2}$ ) of all sunspots according to the routine observations of the solar patrol. We estimate the sum

$$
\mathrm{H}_{\mathrm{s}}=0.15 \underset{i}{\Sigma \mathrm{S}_{i} \mathrm{H}_{i} / \pi \mathrm{R}_{\odot}^{2}}
$$

where $\mathrm{S}_{i}$ is the area of the $i$ th sunspot, including penumbra, having the measured maximal absolute value of field strength $H_{i}$ inside the umbra, and the factor 0.15 is the mean ratio of umbra to penumbra areas. The total flux from the sunspots is often nearly zero, and in those cases where it has an appreciable magnitude there is a considerable tendency for the sunspot flux to be of the opposite polarity to the mean solar field and the interplanetary field. This is consistent with a discussion by Wilcox (1968) that the strong fields of active regions do not contribute directly to the interplanetary magnetic field.

The magnitudes of the mean solar field and of the interplanetary field are compared in Figure 2. The solar field is plotted positive when it is directed out of the sun and negative when it is directed into the sun. The interplanetary field is plotted positive when it is part of a sector with field polarity away from the sun and is plotted negative when it is part of a toward-the-sun sector. Daily observations of the solar field and 3-hour average observations of the interplanetary field are plotted. Most interplanetary sector boundaries are contained within single 3-hour averages so that the interplanetary field magnitude changes abruptly at a sector boundary on this time scale. The change of 
polarity in the solar observations is more gradual since they represent an average of the field on the slowly (on this time scale) rotating solar disk. We note, however, that the magnitude and the sign of the mean solar field can change very rapidly. This change can be as large as approximately one gauss per day, as for example on April 5-6, May 14-15 and June 2-3 (see Figure 1). This is consistent with the earlier results by Severny (1967) relating to the mean polar fields of the sun, since rapid changes of polar fields may be related to rapid changes of fields in other areas contributing to the mean solar field.

We should expect to find the best comparisons of magnitudes in the middle portions of large sectors, and Figure 2 shows that this is usually the case. The scaling shown in the labels of Figure 2 has been chosen to make the average amplitudes of the two fields in the middle portions of large sectors approximately coincide on the graph. Let us see if this scaling is reasonable. The average interplanetary field magnitude of $6 \times 10^{-5}$ gauss corresponds to an average radial component of this field of about $4 \times 10^{-5}$ gauss, since the average Archimedes spiral angle near the earth is about $45^{\circ}$. A radial component of $4 \times 10^{-5}$ gauss in the interplanetary field observed near the earth scales as the radius squared to about 1.8 gauss at the surface of the sun. This field strength must now be multiplied by a factor of about 0.7 for an assumed radial photospheric field or about 0.5 for an assumed semi-isotropic photospheric field, because the observed mean field is the average of the line-of-sight field components from all the elements of area of the solar disk. Thus the scaling yields a solar field magnitude of 1.3 gauss to 0.9 gauss. From the ordinates of Figure 2 we see that the $6 \times 10^{-5}$ gauss average interplanetary field magnitude is scaled to 0.75 gauss for the mean solar field 
magnitude. The approximate scaling we have done has yielded agreement within less than a factor of two of this value.

The close agreement between the observed scaling and the calculated scaling suggests that large areas on the sun (mostly outside of active regions) have a field whose predominant polarity agrees with the polarity of the interplanetary magnetic sector pattern. The distribution with latitude of the photospheric field averaged over all longitudes indicates that the contributions of the low latitude regions $\left(|\theta| \leq 30^{\circ}\right)$ and of the high latitude regions $\left(|\theta|>30^{\circ}\right)$ can be comparable (Severny, 1967, Fig. 14). The further observation of the distribution over the visible disk of the regions responsible for the mean field strength measured for the sun as a star would be very important.

The average time interval between the observation of a polarity change in the mean solar field and the observation of the corresponding interplanetary sector boundary near the earth can be determined by computing a cross-correlation of the two sets of observations as a function of lag. The result of this cross-correlation is shown in Figure 3, in which a positive lag corresponds to the observation of a magnetic feature first on the sun and later near the earth. The centroid of the peak correlation is at a lag of $4 \frac{1}{2} \pm \frac{1}{4}$ days, which is consistent with earlier magnetic field comparisons summarized in wilcox (1968) and with the transit time of solar wind plasma from sun to earth based on observed solar wind velocities.

An interesting effect appears if the cross-correlation described above is extended to lags corresponding to several solar rotations. The result of this cross-correlation is shown in Figure 4. The peak shown in'Figure 3 at a lag of $4 \frac{1}{2}$ days is visible, corresponding to the direct 
transport of magnetic field lines from the sun to the earth. The interplanetary sector pattern usually changes by only a small amount from one solar rotation to the next (see Figure 5), and therefore in Figure 4 we may expect to see other peaks in the cross-correlation at lags separated by multiples of 27 days from the $4 \frac{1}{2}$ day peak. As we move farther away from the $4 \frac{1}{2}$ day peak the magnitude of the correlation peak will decrease since there is a slow evolution of the interplanetary sector pattern with time. This effect is shown by the straight lines drawn through the tops of the shaded peaks in Figure 4. (Ignore for the moment the unshaded peaks; they will be discussed later.) A conspicuous exception to this expected behavior is seen at the peak at a lag of $4 \frac{1}{2}+27$ days. This peak is considerably larger than would be expected; in fact the absolute magnitude of this peak is larger than the magnitude of the peak at $4 \frac{1}{2}$ days. The explanation for the anamolous size of the peak at $4 \frac{1}{2}+27$ days may be found in the discussion of Schatten et al. (1968) showing that there may be a delay of approximately one solar rotation between the appearance of a new magnetic feature in the photosphere and the resulting change in the interplanetary sector pattern. The result of such a delay on the correlation analysis shown in Figure 4 may be understood in the following way. Assume first that the solar magnetic field is stationary in time. Then the resulting interplanetary magnetic sector pattern would also be stationary in time, and an analysis such as that of Figure 4 would show a series of correlation peaks every 27 days all having the same height. Let us now change the polarity of a particular area in the photosphere. In accordance with the above discussion we will assume that there is not a prompt corresponding change in the interplanetary 
sector pattern. Then the correlation peak at a lag of $4 \frac{1}{2}$ days will be somewhat reduced, corresponding to this discrepancy in the direction of the two fields. We may further assume that if we wait for one solar rotation the corresponding change in the interplanetary sector pattern will have occurred, and similarly the correlation at $4 \frac{1}{2}+27$ days will be back to the full value.

We may consider the meaning of the unshaded correlation peaks in Figure 4. Figure 5 shows the interplanetary magnetic sector structure observed during 1968. Sectors with field polarity away from the sun have a light shading and those with field polarity toward the sun have a dark shading. The interval of time during which the interplanetary field was compared with the mean solar field is indicated by the letters labelled SEVERNY in Figure 5. The cross-correlation in Figure 4 is equivalent to comparing the interval in Figure 5 labelled SEVERNY with the other intervals of Figure 5. We may notice that an approximately $180^{\circ}$ phase change has occurred in Figure 5 between the first few rotations of the year and the last few rotations of the year. In the first rotations the first portion of each rotation has field away from the sun, while in the last few rotations most of the first portion of each rotation has field directed toward the sun. This phase shift through the year has caused the unshaded peaks that appear in Figure 4. We may further note from Figure 5 that usually the interplanetary sector pattern changes by only a small amount from one solar rotation to the following rotation.

It is possible by comparing the observed solar and interplanetary magnetic fields to make an independent determination of the zero level of the solar magnetograph. This determination is of some interest, particularly since large areas of the photosphere sometimes are observed to 
have a single dominant polarity. The principle of this determination is to take the zero level of the observed interplanetary field as a standard, since it is known to within about 4 percent of the average field magnitude. We wish to determine whether the addition of a small increment of field $\Delta B$ to the observed mean photospheric field will improve the agreement between the two sets of observations. For this determination a number of crosscorrelations between the average photospheric field magnitude and the interplanetary field magnitude were computed. For each such correlation a small increment of field $\Delta B$ was added to the observed mean photospheric field. Figure 6 shows the peak cross-correlation between the two fields as a function of $\Delta B$. The best agreement between the two sets of observations is obtained by adding $0.1 \pm 0.05$ gauss to the solar observations. This independent determination is consistent with the accuracy of \pm 0.15 gauss quoted by Severny (1970).

During the interval in 1968 examined in this report the mean solar field displayed a predominance toward southern (inward) polarity, both in magnitude and duration (see Figure 1). The reality of this predominance is supported by the agreement between the mean solar field and the interplanetary field. Other examples of the predominance of a single polarity in the solar field for several consecutive rotations have been reported by Severny (1967) and by Wilcox (1970). The physical structure responsible for these interesting observations is a challenging problem. The comparisons discussed here appear to be consistent with a previously discussed pattern in the large-scale weak photospheric magnetic field. Figure 7 shows a sector boundary in this field computed from observations during 1965. To the east of the boundary the field direction 
was predominantly out of the sun and to the west of the boundary the field direction was predominantly into the sun. The solar sector boundaries rotated in an almost rigid system since they were not sheared by the effects of the differential rotation. The agreement between solar and interplanetary fields reported here could exist only if the solar source of the interplanetary field were part of such a field pattern that was ordered over a large portion of the solar disk, as discussed above for Figure 2 in the scaling of the field magnitudes from the sun to near the earth. If the large-scale photospheric field patterns that contribute to the observed mean solar field were subject to a differential rotation of the magnitude reported by Newton and Nunn (1951), the detailed correspondence shown in Figure 1 between polarity changes in the mean solar field and in the interplanetary field would be obscured. Since the correspondence between polarity changes remains good in all the observations we have compared we conclude that the influence of differential rotation on the mean solar field was not large during the interval we have examined.

The schematic shown in Figure 7 was obtained from cross-correlations between the photospheric field observed in the conventional manner with a solar magnetograph and the observed interplanetary field. Figure 8 shows the results of such cross-correlations as obtained by Schatten et al. (1969). The schematic sector boundary of Figure 7 is obtained from Figure 8 in the following manner. We notice in Figure 8 that just to the right of the vertical line corresponding to zero lag there is a positive peak in the correlation. This peak occurs at all the latitudes investigated. Let us assume that at some particular latitude this peak occurs at a lag of $4 \frac{1}{2}$ days, corresponding to the transport by the solar wind plasma of solar magnetic 
field lines from the sun to the interplanetary medium near the earth. The presence of a similar cross-correlation peak at some other heliographic latitude indicates that at this other latitude there exists a photospheric field pattern similar to that of the source latitude. If the lag of the peak at this second latitude is also $4 \frac{1}{2}$ days then a sector boundary would appear at the same longitude at both latitudes. If the lag of the correlation peak at the second latitude were for example $5 \frac{1}{2}$ days then the sector boundary at that latitude would appear at a longitude about $13^{\circ}$ to the west of its position at the source latitude. Thus the relative longitude of the sector boundary shown in Figure 7 as a function of latitude corresponds to the lag of the cross-correlation peak in Figure 8 as a function of latitude. Figure 8 shows the same property that appeared in the present investigation in the discussion of Figure 4, namely that the height of the crosscorrelation peak at a lag of $4 \frac{1}{2}+27$ days is larger than the height of the peak at a lag of $4 \frac{1}{2}$ days. In Figure 8 this property can be seen in each of the latitudes investigated. As discussed above, this property appears to be related to a lag of about one solar rotation between the appearance of a new photospheric magnetic feature and the corresponding change in the interplanetary magnetic sector pattern.

The present investigation has shown that the observed mean solar field is very similar to the interplanetary magnetic field that will be observed near the earth approximately $4 \frac{1}{2}$ days later. Thus the observed mean solar field is a prediction of the interplanetary field that will be observed at the earth 4 or 5 days later, including a prediction of the arrival time of sector boundaries at the earth. The average geomagnetic response to the arrival of a sector boundary has been shown (Wilcox and Colburn, 1969) to 
consist of a monotonic decline in activity in the days preceding the boundary followed by an abrupt increase near the arrival of the boundary. Thus observation of the mean solar field may aid in predicting geomagnetic activity. Several properties of the comparison between the observed mean solar field and the observed interplanetary field appear to be consistent with previous analyses of the solar and interplanetary magnetic sector patterns.

\section{Acknowledgements}

J. M. Wilcox thanks A. Severny for kind hospitality during a visit to the Crimean Astrophysical Observatory.

This work was supported in part by the Office of Naval Research under contract NO0014-69-A-0200-1016, by the National Aeronautics and Space Administration under grant NGL 05-003-230, and by the National Science Foundation under grant GA-1319. 
Mihalov, J. D., Colburn, D. S., Currie, R. G. and Sonett, C. P.: 1968, J. Geophys. Res. $73,943$.

Newton, H. W. and Nunn, M. L.: 1951, Monthly Notices Roy. Astron. Soc. 111,413 .

Schatten, K. H., Ness, N. F. and Wilcox, J. M.: 1968, Solar Physics 5, 240.

Schatten, K. H., Wilcox, J. M. and Ness, N. F.: 1969, Solar Physics 6, 442. Severny, A.: 1955, Izvest. Krim. Astrofiz. Obs. 15, 31.

Severny, A.: 1967, Izvest. Krim. Astrofiz. Obs. 38, 3.

Severny, A.: 1969, Nature 224, 53.

Severny, A.: 1970, Astrophys. J. 159, I 73.

Wilcox, J. M.: 1968, Space Sci. Rev. 8, 258.

Wilcox, J. M.: 1970, J. Geophys. Res. 75, 2587.

Wilcox, J. M. and Colburn, D. S.: 1969, J. Geophys. Res. 74, 2388.

Wilcox, J. M. and Colburn, D. S.: 1970, J. Geophys. Res. (in press).

Wilcox, J. M., Severny, A. and Colburn, D. S.: 1969, Nature 224, 353. 
Figure Captions

Fig. 1. Top: Mean value of the solar magnetic field (dots) and the polarity of the interplanetary magnetic field (bars). The interplanetary field observations are displaced by $4 \frac{1}{2}$ days to allow for the average transit time of solar wind plasma from the sun to the earth. The abscissa is the time of the solar observations. Bottom: Contribution of sunspot magnetic fields to the mean solar field (after Wilcox et al., 1969).

Fig. 2. Comparison of the magnitude of the mean solar field and of the interplanetary field. The open circles are the daily observations of the mean solar field, and the dots are 3-hour average values of the interplanetary field magnitude observed near the earth. The solar observations are displaced by $4 \frac{1}{2}$ days to allow for the average sun-earth transit time. The abscissa is the time of the interplanetary observations.

Fig. 3. Cross-correlation of the direction of the mean solar field with the direction of the interplanetary field. The centroid of the peak correlation is at a lag of $4 \frac{1}{2} \pm \frac{1}{4}$ days.

Fig. 4. Same as Figure 3, but with the lag of the cross-correlation extended over several solar rotations.

Fig. 5. Interplanetary magnetic sector structure observed during 1968 with Explorers 33 and 35 overlayed on a chart of planetary magnetic 3-hourrange indices $\mathrm{Kp}$ (after Bartels). Dark shading is field polarity toward the sun and light shading is field polarity away from the sun. During the time interval labelled SEVERNY this interplanetary sector pattern was compared with the mean solar field in the present investigation (after Wilcox and Colburn, 1970). 
Fig. 6. Independent determination of the zero level of the solar magnetograph. The peak cross-correlation of the mean solar field with the interplanetary field is plotted as a function of an increment $\triangle B$ added to the observed solar field. The best agreement is obtained when $0.1 \pm 0.05$ gauss is added to the observed solar field.

Fig. 7. Schematic of the average position of a solar sector boundary during 1965. On each side of the boundary the weak background photospheric magnetic field was predominantly of a single polarity in equatorial latitudes on both sides of the equator (after wilcox et al., 1969).

Fig. 8. Cross-correlation of the solar magnetic field calculated on a source surface (see Schatten et al., 1969) $0.5 \mathrm{R}_{\odot}$ above the photosphere with the radial component of the interplanetary magnetic field as a function of time lag. Nine solar rotations of data are utilized, with correlation extending from $45^{\circ} \mathrm{N}$ to $35^{\circ} \mathrm{S}$ in intervals of $5^{\circ}$. The curve near the label $45^{\circ}$ is the cross-correlation between the solar field at that latitude and the interplanetary field, with the horizontal line labelled $45^{\circ}$ representing zero cross-correlation, the horizontal line above representing 1.0 correlation and the horizontal line next below representing -1.0 correlation. The results for all other latitudes are displaced in a similar format. 


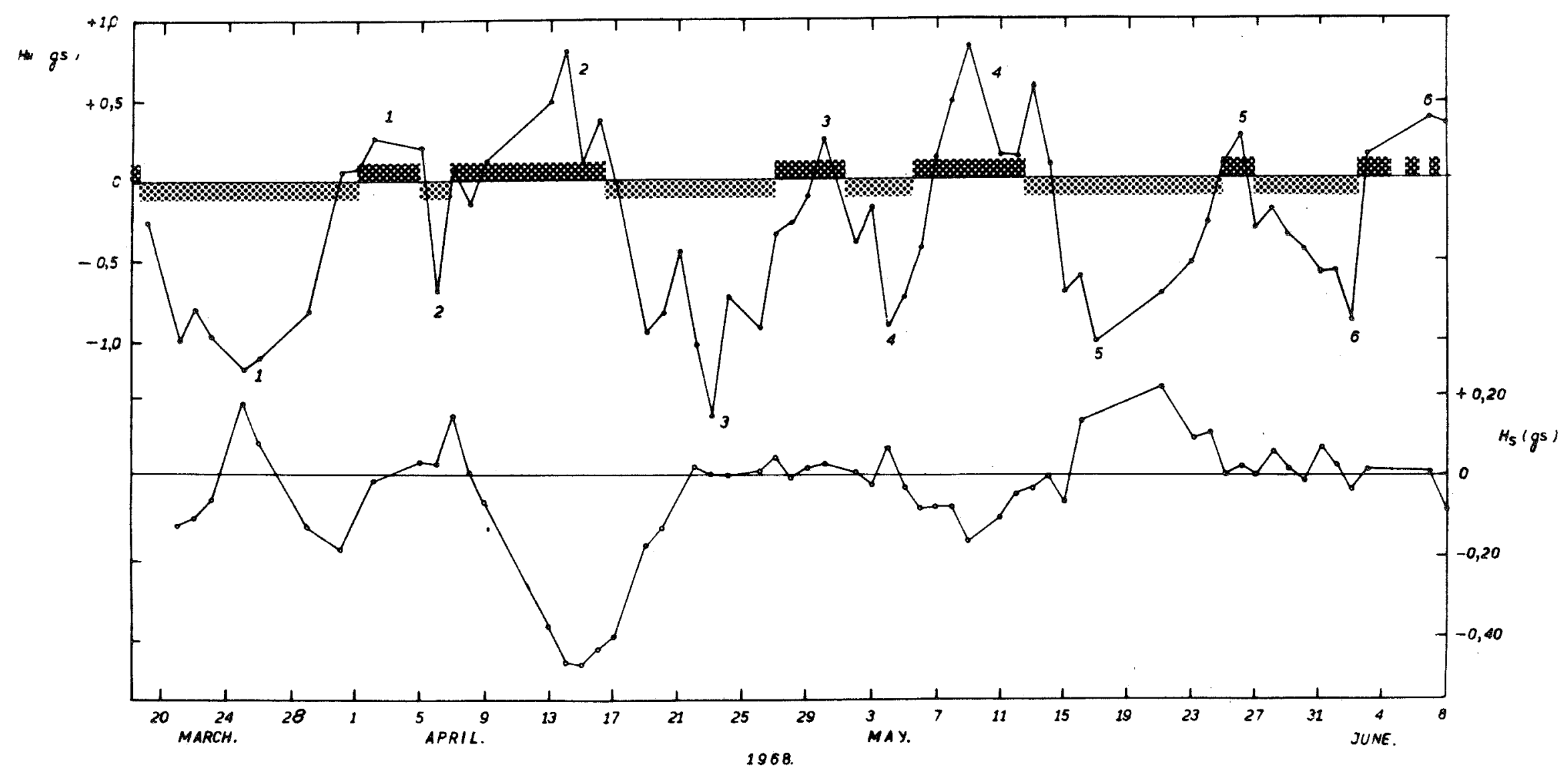


INTERPLANETARY FIELD MAGNITUDE $\left(10^{-5}\right.$ GAUSS $)$

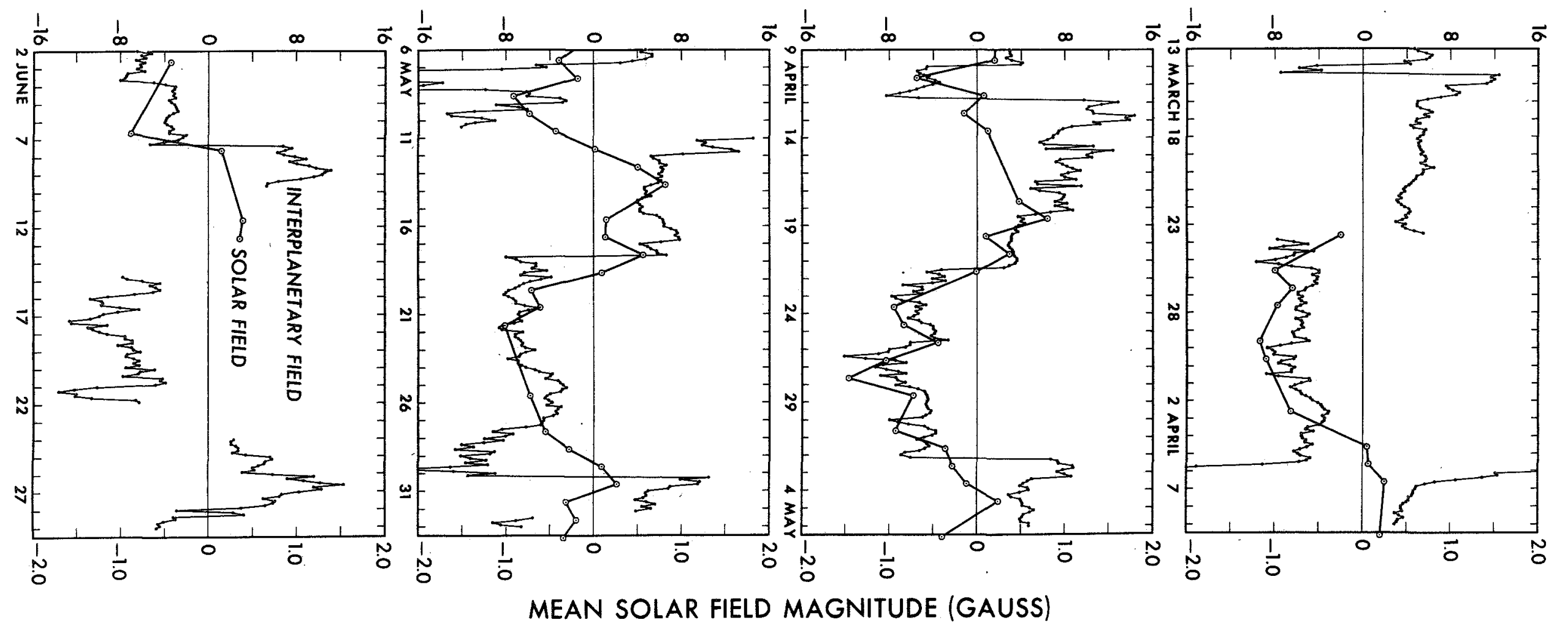


CROSS-CORRELATION OF MEAN SOLAR FIELD WITH INTERPLANETARY FIELD

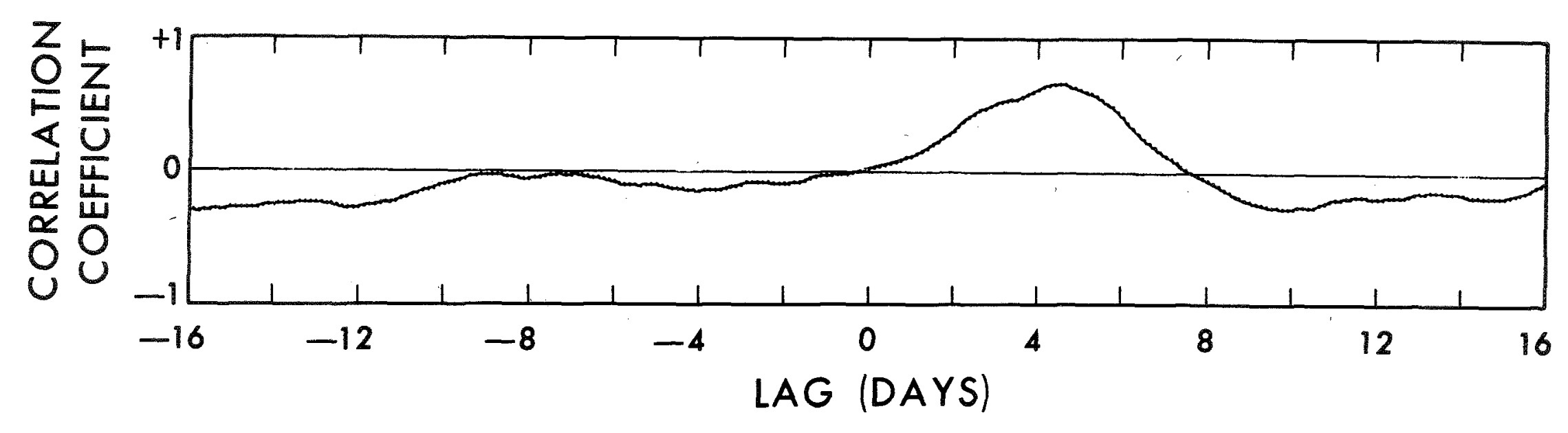


CROSS-CORRELATION OF MEAN SOLAR FIELD WITH INTERPLANETARY FIELD

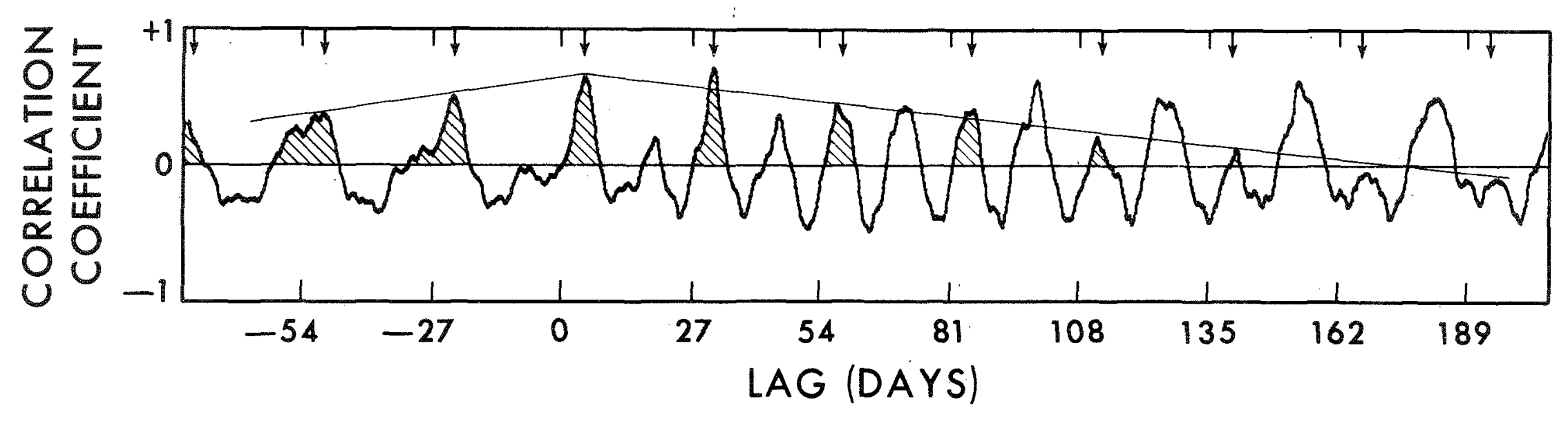




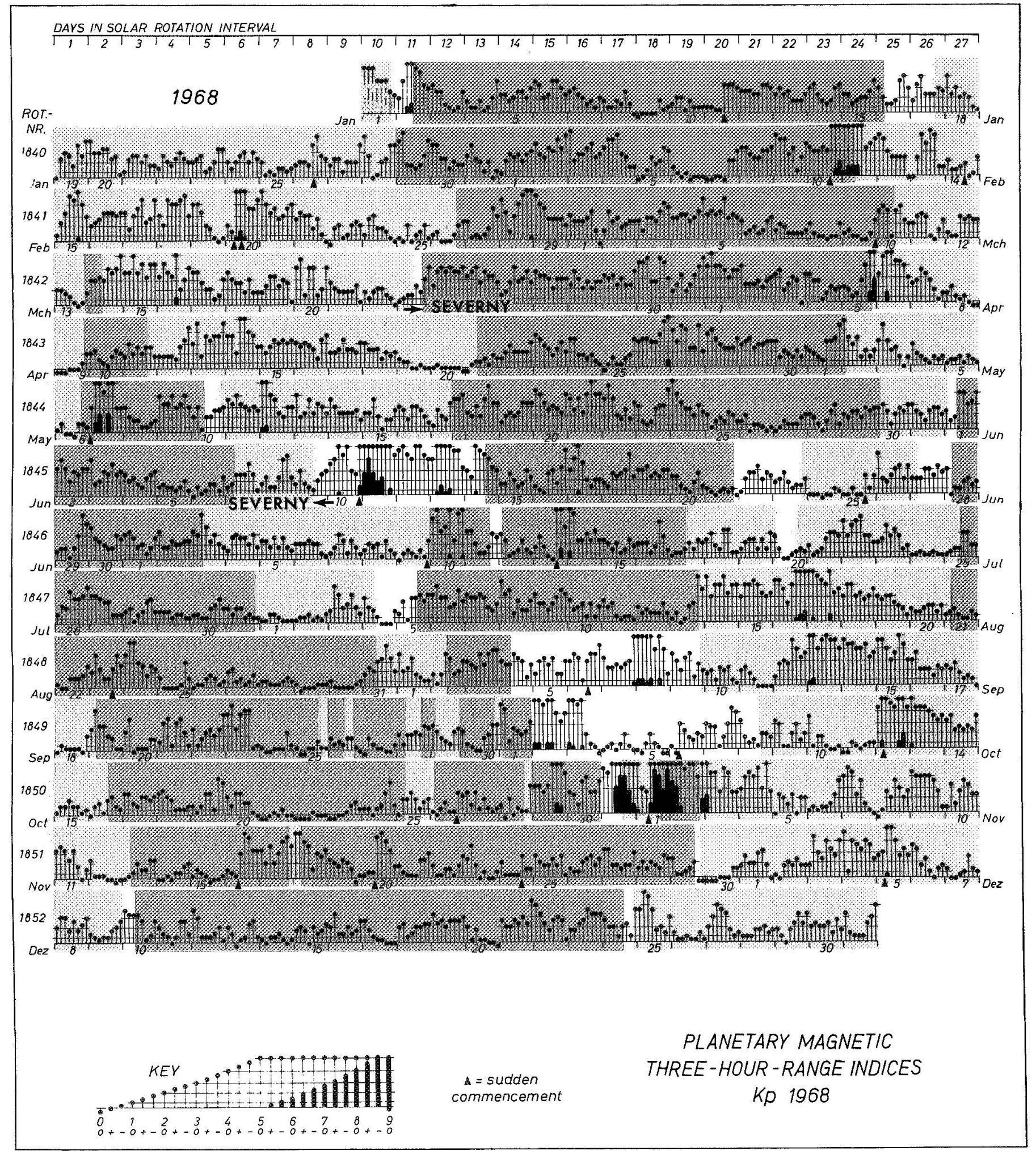

Figure 5 
CROSS CORRELATION OF MEAN SOLAR FIELD WITH INTERPLANETARY FIELD

AS A FUNCTION OF DISPLACEMENT $\triangle B$ OF OBSERVED SOLAR FIELD

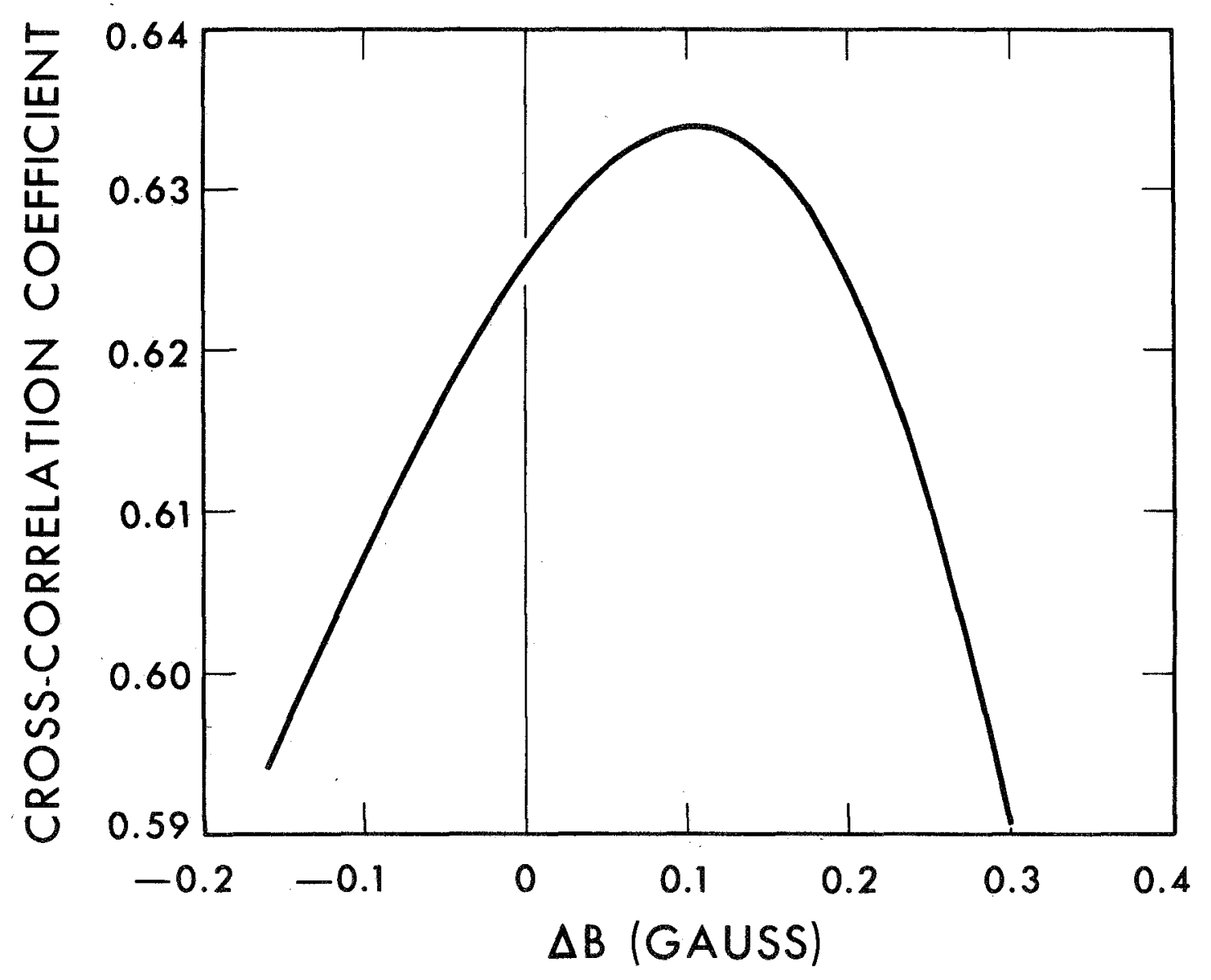

Figure 6 


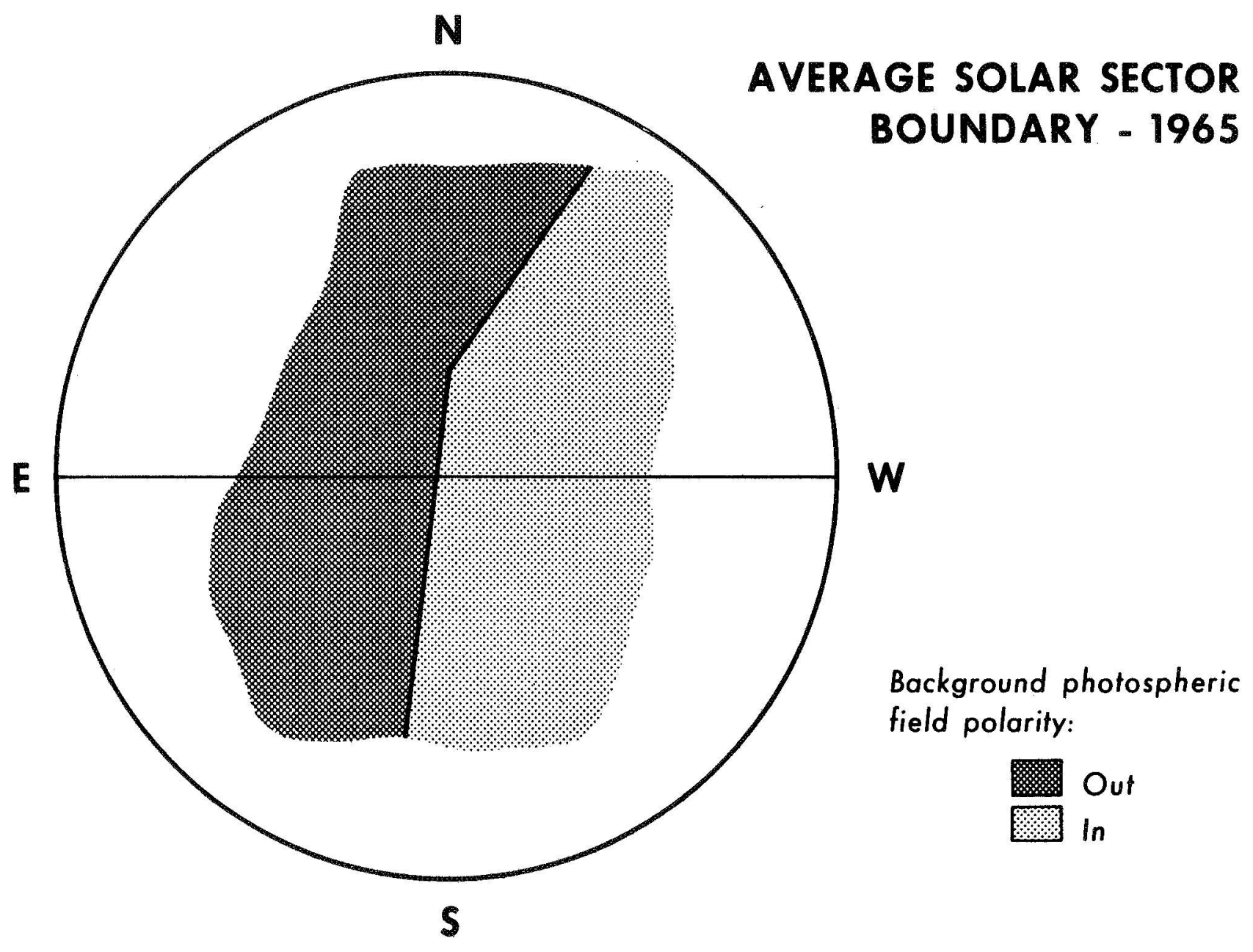


CROSS CORRELATION OF

MAGNETIC FIELD CALCULATED

AT $0.50 R_{\odot}$ ABOVE PHOTOSPHERE

WITH INTERPLANETARY QUANTITY $-B_{r}$

Days $6 / 6 / 65$ to $2 / 4 / 66$

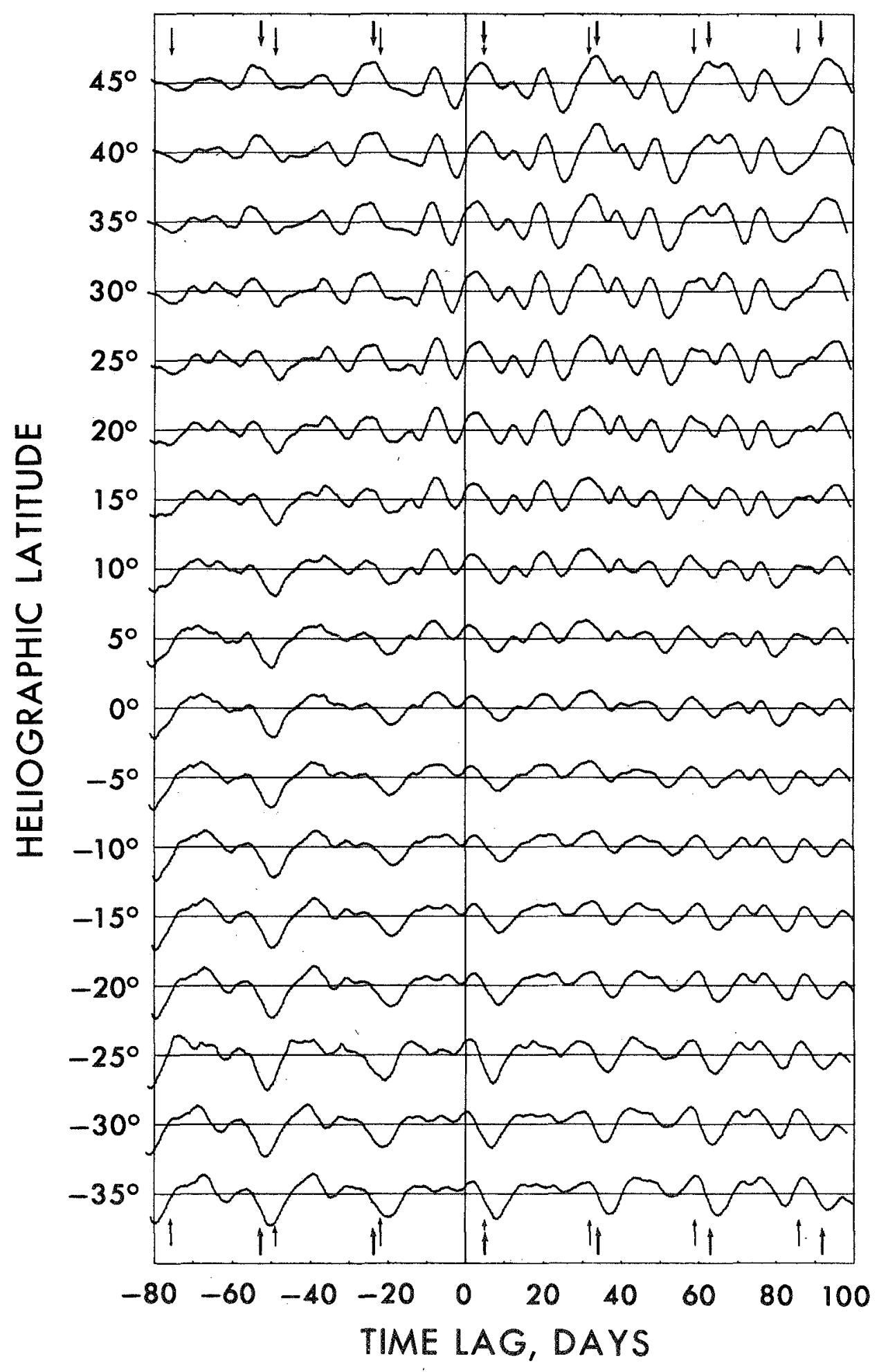

Figure 8 
UNCLASSIFIED

Security Classification

DOCUMENT CONTROL DATA - R\&D

(Soctrity classification of title, body of abstrect and indexing annotation must bu ortered whon the ovorall report is cless ifiod)

1. ORIGINA.TING ACTINITY (Corporato author)

Space Sciences Laboratory

University of California

Berkeley, California 94720

3. REPORT TITLE

COMPARISON OF THE MEAN PHOTOSPHERIC MAGNETIC FIETD AND THE INTERPLANETARY MAGNETIC FIELD

4. DESCRIPTIVE NOTES (Type of roport and inclusive datos)

Technical Report

5. AUTHOR(S) (Last mang, first namo, initial)

Severny, A., Wilcox, John M., Scherrer, Philip H. and Colburn, David S.

\begin{tabular}{|c|c|c|}
\hline $\begin{array}{l}\text { 6. REPO RT DATE } \\
\text { MaY 5, } 1970\end{array}$ & $\begin{array}{l}\text { 7a. TOTALNO. OF PAGES } \\
20\end{array}$ & $\begin{array}{c}\text { 7b. NO. OFREFS } \\
9\end{array}$ \\
\hline 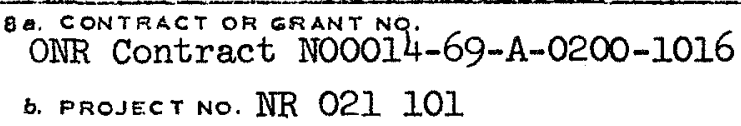 & \multicolumn{2}{|c|}{$\begin{array}{l}\text { 9. ORIGINATOR'S REPORT NUMBER(S) } \\
\text { Series 11, Issue } 36\end{array}$} \\
\hline $\begin{array}{l}\text { c. NASA Grant NGL 05-003-230 } \\
\text { d. NSF Grant GA-1319 }\end{array}$ & \multicolumn{2}{|c|}{$\begin{array}{l}\text { Sb. OTHERREPORT NO(S) (A ny other numbers that may be asslatried } \\
\text { this roport) }\end{array}$} \\
\hline
\end{tabular}

10. AVAILABILITY/LIMITATION NOTICES

Qualified requesters may obtain copies of this report from DDC.

11. SUPPLEMENTARY NOTES

12. SPONSORING MILITARY ACTIVITY

Nuclear Physics Branch

Office of Naval Research

Washington, D. C. 20360

13. ABSTRACT

The mean photospheric magnetic field of the sun seen as a star has been compared with the interplanetary magnetic field observed with spacecraft near the earth.

Each change in polarity of the mean solar field is followed about $4 \frac{1}{2}$ days later by a change in polarity of the interplanetary field (sector boundary). The scaling of the field magnitude from sun to near earth is within a factor of two of the theoretical value, indicating that large areas on the sun have the same predominant polarity as that of the interplanetary sector pattern. An independent determination of the accuracy of the solar magnetograph has yielded a value of $0.1 \pm 0.05$ gauss. An effect attributed to a delay of approximately one solar rotation between the appearance of a new photospheric magnetic feature and the resulting change in the interplanetary field is observed. The present results appear to be consistent W. with preyious investigations.

1) 1 FORA $2: 3$ 
INCTASSTITED

Security Ciassification

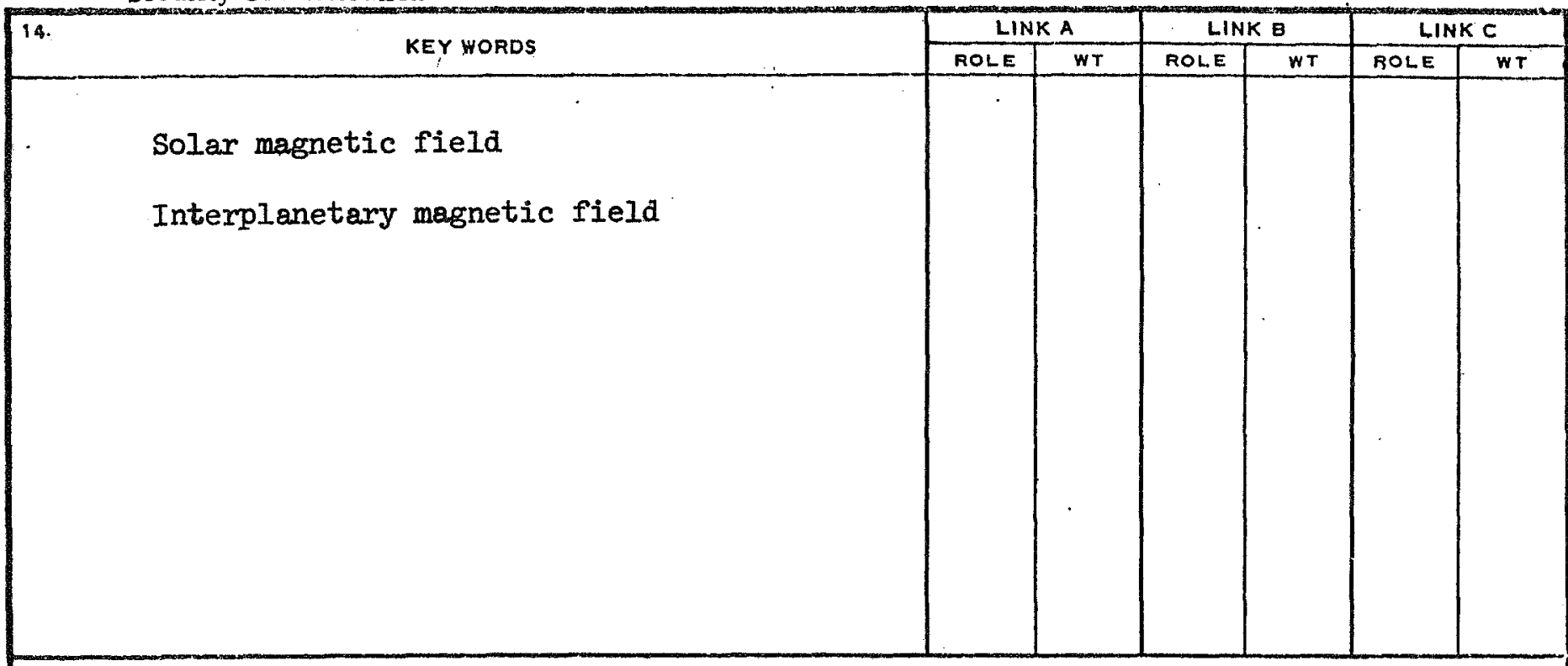

